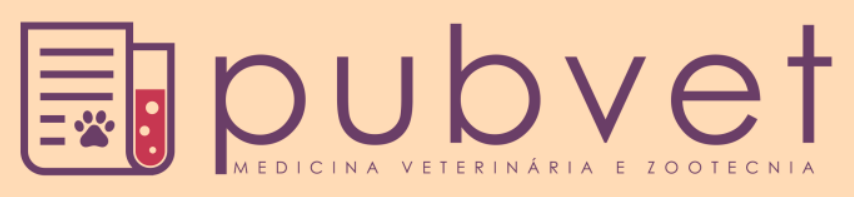

https://doi.org/10.22256/pubvet.v12n7a128.1-4

\title{
Resposta da mandioca a diferentes tipos de preparo do solo
}

\author{
Júlio Cesar Reis Araújo1, Alandionys Gonçalves de Lima², Juracy Mendes Moreira ${ }^{30}$, \\ Antonio Florentino de Lima Junior ${ }^{4}{ }^{\circ}$, Arinaldo Pereira da Silva ${ }^{5} \bullet$, Lucas Roberto de \\ Carvalho $^{50}$, Juliano Queiroz Santana Rosa ${ }^{6 *}$
}

${ }^{1}$ Engenheiro Agronômico, E-mail: alandionysgl@ gmail.com

${ }^{2}$ Engenheiro Agronômico, E-mail: jc-reisaraujo@hotmail.com

${ }^{3}$ Prof. Faculdade Montes Belos. São Luiz de Montes Belos-GO. E-mail: juraci.moreira@fmb.edu.br

${ }^{4}$ Prof. Faculdade Montes Belos. São Luiz de Montes Belos - GO. E-mail: agronomia@fmb.edu.br

${ }^{5}$ Prof. Faculdade Montes Belos. São Luiz de Montes Belos - GO. E-mail: arinaldo.pereira@fmb.edu.br

${ }^{6}$ Prof. Faculdade Montes Belos. São Luiz de Montes Belos - GO. E-mail: lucas.roberto@fmb.edu.br

${ }^{7}$ Prof. Faculdade Montes Belos. São Luiz de Montes Belos - GO. E-mail: julianoqsr@gmail.com. *Autor para correspondencia.

\begin{abstract}
RESUMO. A mandioca (Manihot esculenta Crantz) é uma espécie nativa do Brasil central presente em todos os territórios tropicais e subtropicais, tem boa adaptabilidade, tolerância a seca e apresenta rendimento satisfatório em solos de baixa fertilidade, cultura promissora na agricultura familiar, contribuindo no desenvolvimento social e econômico do Brasil. Apesar disso, tradicionalmente a cultura é conduzida com baixo investimento financeiro, geralmente, não havendo aplicação de corretivos e fertilizantes em doses adequadas. $\mathrm{O}$ objetivo deste trabalho foi avaliar o efeito de dois tipos de preparo de solo; realização ou não de gessagem; e quatro doses de calcário na produtividade da mandioca (IAC 12). O experimento foi desenvolvido em parcela subsubdividida com 24 repetições. Avaliaram-se dois tipos de preparo de solo um com arado e grade e o outro somente grade; gessagem (0 e $650 \mathrm{~kg} / \mathrm{ha})$; e quatro doses de calcário $(0 ; 600 ; 1000 ; 1400 \mathrm{~kg} / \mathrm{ha})$. A colheita foi realizada 10 meses após o plantio e foi determinada a produtividade por planta. Foi realizada análise de variância e teste de Scott-Knott para comparação de médias. Verificou-se que não houve resposta significativa para nenhum dos tratamentos realizados.
\end{abstract}

Palavras chave: Cultivo, gessagem, calagem, produtividade

\section{Response of cassava to different types of soil preparation}

ABSTRACT. Manihot esculenta Crantz is a native species of central Brazil present in all tropical and subtropical territories, has good adaptability, tolerance to drought and presents satisfactory yield in low fertility soils, promising crop in the family agriculture, contributing to the social and economic development of Brazil. In spite of this, traditionally, the culture is conducted with low financial investment, and in general, does not have the application of corrective and fertilizer in adequate doses. The objective of this work was evaluate the effect of two types of soil preparation; achievement or not of plaster; and four limestone rates on cassava yield (IAC 12). The experiment was carried out in a sub-divided plot with 24 replicates. Two types of soil preparation were evaluated: one with plow and grating and the other only with grating; plastering ( 0 and $650 \mathrm{~kg} / \mathrm{ha})$; and four doses of limestone $(0,600,1000,1400 \mathrm{~kg} / \mathrm{ha})$. Harvesting was performed 10 months after planting and productivity per plant was determined. Variance analysis and ScottKnott's test were performed to compare means. It was found that there was no significant response to any of the treatments performed.

Keywords: Cultivation, plastering, liming. productivity 


\section{Respuesta de la Manihot esculenta Crantz a diferentes tipos de preparación del suelo}

RESUMEN. La yuca (Manihot esculenta Crantz) es una especie nativa del Brasil central presente en todos los territorios tropicales y subtropicales, tiene buena adaptabilidad, tolerancia a la sequía y presenta rendimiento satisfactorio en suelos de baja fertilidad, cultivo promisorio, en la agricultura familiar, contribuyendo en el desarrollo social y económico de Brasil. A pesar de ello, tradicionalmente el cultivo es conducido con poca inversión financiera, generalmente, sin aplicación de correctivos y fertilizantes a dosis adecuadas. El objetivo de este trabajo fue evaluar el efecto de dos tipos de preparación de suelo; realización o no de yeso; y cuatro dosis de cal en el rendimiento productivo de la yuca (IAC 12). El experimento fue realizado en parcela subsubdividida con 24 repeticiones. Se evaluaron dos tipos de preparación de suelo uno con arado y alambrado y el otro sólo alambrado; aplicación de yeso (0 y $650 \mathrm{~kg} / \mathrm{ha}$ ); y cuatro dosis de cal $(0,600,1000,1400$ $\mathrm{kg} / \mathrm{ha}$ ). La cosecha se realizó 10 meses después de la siembra y se determinó la productividad por planta. Se realizó un análisis de varianza y prueba de Scott-Knott para la comparación de promedios. Se verificó que no hubo respuesta significativa para ninguno de los tratamientos realizados.

Palabras clave: Cultivo, aplicación de yeso, calado, productividad

\section{Introdução}

A mandioca (Manihot esculenta Crantz), juntamente com o milho, a cana-de-açucar e arroz, constituem as fontes de energia mais importantes das regiões tropicais do mundo, originaria da América do Sul, Tironi et al. (2015). A cultura da mandioca desde o Brasil colonial sempre foi a base econômica de subsistência dos agricultores familiares, historicamente esteve presente nas conquistas do sertão, Oliveira et al. (2012). A mandioca é o tubérculo especificamente mais difundido do gênero manioht, composto por várias raízes comestíveis,

A mandioca encontra ambiente propício para o seu desenvolvimento em climas tropicais e subtropicais. Cultivada na faixa entre 30 graus de latitudes Norte e Sul, embora o plantio da mandioca esteja mais localizado entre as latitudes $20^{\circ} \mathrm{N}$ e $20^{\circ} \mathrm{S}$, ela vai bem em altitudes, desde o nível do mar até aproximadamente 2.300 metros, porém regiões com altitudes de até 600 a 800 metros são as mais adequadas, a temperatura média ideal está entre os 20 a $27^{\circ} \mathrm{C}$, podendo a planta crescer bem entre 16 e $38^{\circ} \mathrm{C}$, temperaturas em torno de $15^{\circ} \mathrm{C}$ retardam a germinação ou paralisa sua atividade vegetativa, Otsubo \& Lorenzi (2002).

A mandioca é bem tolerante às condições de acidez dos nossos solos, mas bastante sensível a alto $\mathrm{pH}$ e a solos salinos, não sendo observado aumento significativo da produção com doses mais elevadas de calcário, Távora et al. (2002). O nível de saturação de bases a ser adotado deverá ser de $40 \%$, não ultrapassando a 2 toneladas por hectare, e de calcário PRNT 100\%, quantidades excessivas de calcário podem ocasionar deficiência de zinco e manganês nas plantas Takahashi \& Guerini (2008). Assim como a calagem a adubação também deve ser baseada nos níveis de nutrientes observados na análise de solo.

\section{Material e Métodos}

$\mathrm{O}$ experimento foi realizado em Americano do Brasil, (Goiás) no período de 08/2016 a 11/2017, (latitude: $16^{\circ} 15^{\prime} 17^{\prime}$ ' S; longitude: $49^{\circ} 58^{\prime} 58^{\prime}$ ' W); situada a uma altitude de $890 \mathrm{~m}$. O experimento foi desenvolvido em um Latossolo Vermelho de textura média, segue abaixo na Tabela 1 a análise química do solo.

Tabela 1. Análise de solo da área do experimento. Americano do Brasil, 2017.

\begin{tabular}{|c|c|c|c|c|c|c|c|c|c|c|c|c|}
\hline Prof & $\mathrm{pH}$ & M.O & $\mathrm{P}$ & $\mathrm{K}$ & $\mathrm{Ca}$ & $\mathrm{Mg}$ & $\mathrm{Al}$ & $\mathrm{H}+\mathrm{Al}$ & Arg & $\mathrm{m}$ & CTC & V \\
\hline $\mathrm{Cm}$ & & & \multicolumn{2}{|c|}{$\mathrm{mg} / \mathrm{dm}^{3}$} & & \multicolumn{3}{|c|}{$\mathrm{cmolc} / \mathrm{dm}^{3}$} & & $\%$ & $\%$ & $\%$ \\
\hline $0-20$ & 5,2 & 0,70 & 1,44 & 46,0 & 0,88 & 0,13 & 0,10 & 2,10 & 26 & 8,16 & 3,23 & 34,6 \\
\hline $20-40$ & 5,2 & 0,60 & 0,81 & 20,0 & 0,63 & 0,50 & 0,15 & 2,10 & 30 & 11,2 & 3,28 & 35,8 \\
\hline
\end{tabular}


$\mathrm{O}$ experimento foi instalado em delineamento experimental em parcela subsubdividida com 24 repetições, e desenho experimental inteiramente casualizado. Cada parcela (repetição) foi constituída de 3 linhas com 10m de comprimento. O espaçamento de plantio foi de $0,9 \mathrm{~m}$ entre linhas e 0,6 metros entre plantas. Segue abaixo descrição dos tratamentos analisados:

- Parcela Principal (Preparo de solo, com dois fatores):

1) arado + grade

2) grade

- Subparcela (Gessagem, com dois fatores):

1) Sem gesso

2) Com $650 \mathrm{~kg} / \mathrm{ha}$ de gesso

- Subsubparcela (Calagem, com quatro doses):

1) Sem calagem

2) $600 \mathrm{~kg} / \mathrm{ha} \mathrm{de} \mathrm{calcário}$

3) $1000 \mathrm{~kg} / \mathrm{ha} \mathrm{de} \mathrm{calcário}$

4) $1400 \mathrm{~kg} / \mathrm{ha}$ de calcário

O preparo do solo foi realizado em 09/10/2016. Sendo que o calcário foi aplicado em 14/10/2016, em seguida foi incorporado ao solo com grade niveladora. O gesso foi aplicado em 16/11/2016, incorporado em seguida ao solo também com grade niveladora. O plantio foi realizado em 12/12/2016, ou seja, dois meses após aplicação do calcário e um mês após aplicação do gesso. A recomendação para a dose do gesso foi realizada conforme a Ribeiro et al. (1999) e a análise do solo (Tabela 1). Para estabelecimento das doses de calcário entaleceu-se a quantidade necessária para atingir a saturação de bases de 50\%, 60\% e $70 \%$ respectivamente, tendo em vista que está faixa de $50 \%$ a $70 \%$ é considerada a mais recomendada para a maioria das culturas no cerrado.

No plantio foi aplicado supersimples na mesma dose em todas as parcelas, seguindo recomendação de Sousa \& Lobato (2004). No dia 19/02/2017 foi realizada cobertura com 20-00-20. Durante a condução da lavoura foi realizado controle de plantas daninhas com herbicidas pós emergentes em jato dirigido.

A colheita foi realizada em 23/10/2017, ou seja, 10 meses após o plantio. Foi realizada pesagem da massa fresca das raízes e contagem do número de plantas em cada parcela, calculando-se produtividade média por planta. Os dados foram submetidos à análise variância utilizando o Software estatístico R ( $\underline{\mathrm{R} \text { Development Core }}$ Team, 2017).

\section{Resultados e Discussão}

Conforme observado na Tabela 2 a cultura não respondeu ao preparo de solo mais profundo, indicando que em condições normais de cultivo e solo o produtor pode usar apenas o preparo convencional com grade aradora, o que pode reduzir custos.

Com relação ao Gesso, observou-se que a cultura não respondeu a aplicação (Tabela 3). Então, ao menos na análise de uma safra, não foi economicamente interessante a aplicação do Gesso na cultura da mandioca, certamente isto ocorreu pela alta rusticidade da cultura e enorme tolerância a acidez do solo. Resultados semelhantes foram encontrados por Nogueira et al. (1992) em Latossolo roxo de textura argilosa.

Quanto à resposta a calagem (Tabela 4), também foi observada a ausência de resposta as doses de calcário, o que também corrobora com as sugestões da literatura.

Diante dos resultados, percebemos que muita embora tenha havido evolução no melhoramento genético e no perfil produtivo das cultivares de mandioca, a cultura manteve sua alta rusticidade e baixa capacidade de reposta a correção do solo. $\mathrm{Ou}$ seja, os produtores não necessitam de grandes investimentos tanto no preparo, quanto na correção do solo. Fator este positivo e que continua favorecendo o cultivo da mandioca em todas as regiões e pelos mais diversos perfis de produtores rurais.

Tabela 2. Produtividade da mandioca mediante os dois tipos de preparo do solo.

\begin{tabular}{lccc}
\hline Preparo do solo & Grade & Grade+Arado & Cv\% \\
\hline Produtividade kg/planta & $0,847 \mathrm{a}$ & $0,821 \mathrm{a}$ & 30,49 \\
\hline Médias seguidas pela mesma letra não diferiram significativamente pelo teste Scott-Knott a 5\% &
\end{tabular}

Médias seguidas pela mesma letra não diferiram significativamente pelo teste Scott-Knott a 5\%.

Tabela 3. Produtividade da mandioca mediante a presença e ausência de gesso.

\begin{tabular}{lccc}
\hline Gesso & Com & Sem & Cv\% \\
\hline Produtividade kg/planta & $0,830 \mathrm{a}$ & $0,838 \mathrm{a}$ & 30,40 \\
\hline
\end{tabular}

Médias seguidas pela mesma letra não diferiram significativamente pelo teste Scott-Knott a 5\%. 
Tabela 4. Produtividade da mandioca mediante a quatro doses deferentes de calcário.

\begin{tabular}{lccccc}
\hline Calcário & $0 \mathrm{~kg} / \mathrm{ha}$ & $600 \mathrm{~kg} / \mathrm{ha}$ & $1000 \mathrm{~kg} / \mathrm{ha}$ & $1400 \mathrm{~kg} / \mathrm{ha}$ & $\mathrm{CV} \%$ \\
\hline Produtividade kg/planta & $0,848 \mathrm{a}$ & $0,852 \mathrm{a}$ & $0,829 \mathrm{a}$ & $0,806 \mathrm{a}$ & 30,49 \\
\hline
\end{tabular}

Médias seguidas pela mesma letra não diferiram significativamente pelo teste Scott-Knott a 5\%.

\section{Conclusão}

A mandioca IAC 12 não respondeu ao preparo mais profundo do solo e nem a correção do solo com Gesso e Calcário. Confirmando a rusticidade e a tolerância à acidez por parte da planta.

\section{Referências Bibliográficas}

Nogueira, F. D., Paula, M. B., Gontijo, P. T. G. \& Tanaka, T. 1992. Adubação verde, fosfato natural e gesso para a cultura da mandioca em Latossolo roxo textura argilosa. Pesquisa. Agropecuária. Brasileira, 27, 357-372.

Oliveira, T. O., Uchôa, S. C., Alves, J. M. \& Sediyama, T., Albuquerque, J. A. A., Souza, E. D. \& Melville, C. C. 2012. Ácido cianídrico em tecidos de mandioca em função da idade da planta e adubação nitrogenada. Pesquisa Agropecuária Brasileira, 47(10), 1436-1442.

Otsubo, A. A. \& Lorenzi, J. O. 2004. Cultivo da mandioca na Região Centro-Sul do Brasil. Embrapa Agropecuária, BR.

$\mathrm{R}$ Development Core Team: A language and environment for statistical computing. 2014. R Foundation for Statistical Computing, versão 2.13.1. Vienna, Austria. Disponível em: $<$ http://www.R-project.org $>$. Acesso em: $19 / 01 / 18$

Ribeiro, A. C., Guimarães, P. T. G. \& Alvarez, V. V. H. 1999. Comissão De Fertilidade De Solos Do Estado De Minas Gerais. Recomendações para o uso de corretivos e fertilizantes em Minas Gerais 359 p.). CFSEMG, Viçosa, M. G., BR.

Sousa, D. M. G. \& Lobato, E. 2004. Cerrado: correção do solo e adubação ( $2^{\mathrm{a}}$ ed., 416 p.). EMBRAPA - Informação tecnológica, Brasília, DF, BR.

Takahashi, M. \& Guerini, V. L. 2008. Espaçamento para a cultura da mandioca. Brazilian Archives of Biology and Technology, 14(4), 489-494.

Távora, F. J. A. F., Queiroz, G. M. de, Pinho, J. L. N. de \& Melo, F. I. O. 2002. Comportamento de cultivares de mandioca com diferentes características foliares submetidas a diversas densidades populacionais. Pesquisa Agropecuária Brasileira, 17(3), 417-431.

Tironi, L. F., Uhlmann, O. L., Streck, N. A. \& Samboranha, F. K. 2015. Desempenho de cultivares de mandioca em ambiente subtropical, Bragantia, Campinas, 74(1), 5866.

\section{Article History:}

Received 15 March 2018

Accepted 13 April 2018

Available online 9 June 2018

License information: This is an open-access article distributed under the terms of the Creative Commons Attribution License 4.0, which permits unrestricted

use, distribution, and reproduction in any medium, provided the original work is properly cited. 